\title{
Response to Targeted Cognitive Training Correlates with Change in Thalamic Volume in a Randomized Trial for Early Schizophrenia
}

\author{
Ian S Ramsay*, , Susanna Fryer ${ }^{2,3}$, Alison Boos ${ }^{2}$, Brian J Roach ${ }^{2,3}$, Melissa Fisher ${ }^{1,2}$, Rachel Loewy ${ }^{2}$, \\ Sophia Vinogradov ${ }^{1,2,3}$ and Daniel H Mathalon ${ }^{2,3}$ \\ 'Department of Psychiatry, University of Minnesota, Minneapolis, MN, USA; '2Department of Psychiatry, University of California, San Francisco, CA, \\ USA; ${ }^{3}$ San Francisco VA Medical Center, San Francisco, CA, USA
}

\begin{abstract}
Reduced thalamic volume is consistently observed in schizophrenia, and correlates with cognitive impairment. Targeted cognitive training (TCT) of auditory processing in schizophrenia drives improvements in cognition that are believed to result from functional neuroplasticity in prefrontal and auditory cortices. In this study, we sought to determine whether response to TCT is also associated with structural neuroplastic changes in thalamic volume in patients with early schizophrenia (ESZ). Additionally, we examined baseline clinical, cognitive, and neural characteristics predictive of a positive response to TCT. ESZ patients were randomly assigned to undergo either $40 \mathrm{~h}$ of TCT $(N=22)$ or a computer games control condition (CG; $N=22 \mathrm{~s}$ ). Participants underwent MRI, clinical, and neurocognitive assessments before and after training (4-month interval). Freesurfer automated segmentation of the subcortical surface was carried out to measure thalamic volume at both time points. Left thalamic volume at baseline correlated with baseline global cognition, while a similar trend was observed in the right thalamus. The relationship between change in cognition and change in left thalamus volume differed between groups, with a significant positive correlation in the TCT group and a negative trend in the CG group. Lower baseline symptoms were related to improvements in cognition and left thalamic volume preservation following TCT. These findings suggest that the cognitive gains induced by TCT in ESZ are associated with structural neuroplasticity in the thalamus. Greater symptom severity at baseline reduced the likelihood of response to TCT both with respect to improved cognition and change in thalamic volume.

Neuropsychopharmacology (2018) 43, 590-597; doi:I0.1038/npp.2017.213; published online I8 October 2017
\end{abstract}

\section{INTRODUCTION}

The thalamus plays a crucial role in the integration of neuronal signals across cortical and subcortical brain areas (Guillery, 1995), and is strongly implicated in both early sensory processing and subsequent higher-order cognitive operations (Gilbert and Sigman, 2007). Pathology of the thalamus has long been implicated in schizophrenia (Cronenwett and Csernansky, 2010; Pergola et al, 2015); meta-analyses demonstrate reduced thalamic volume, among other findings (Ellison-Wright et al, 2008; van Erp et al, 2016; Konick and Friedman, 2001), with some evidence indicating progressive thalamic volume loss over the course of the illness (Haren et al, 2007) possibly related to antipsychotic treatment (Ho et al, 2011). Thalamic volume reductions are also found in healthy relatives of individuals with schizophrenia (Lawrie et al, 2001; Seidman et al, 1999), with recent evidence highlighting specific subthalamic nuclei

*Correspondence: Dr IS Ramsay, Department of Psychiatry, University of Minnesota, 2450 Riverside Ave., Suite F275, Minneapolis, MN 55454, USA, Tel: + 612625 1838, E-mail: ramsa045@umn.edu

Received 27 April 2017; revised 20 August 2017; accepted 5 September 2017; accepted article preview online 6 September 2017 that may be associated with a familial risk for schizophrenia (Pergola et al, 2017). Overall these findings suggest that underlying etiologic factors related to psychosis vulnerability are contributing to thalamic pathology.

Sensory processing and integration is thought to be one of the thalamus' primary roles (Çetin et al, 2014; Morgenstern et al, 2016), and abnormalities in this structure may contribute to psychopathology associated with schizophrenia (Andreasen et al, 1994). Disruptions in sensory processing (Javitt, 2009) are found to have influence on symptoms (Doniger et al, 2001; Yeap et al, 2008) as well as higher-order cognition and functioning (Light et al, 2007), processes that both rely on thalamo-cortical feedback loops (Saalmann and Kastner, 2011). Regional deficits may be characterized by longitudinal volume changes in specific subthalamic nuclei such as the pulvinar, anterior, and medial dorsal nucleus of the thalamus (Cobia et al, 2016); these connectivity-rich thalamic subregions are critical for memory, higher-order cognition, and attention, and were also found to relate to changes in frontal, temporal, and parietal brain areas. However, the etiology of these disruptions remains unclear, as neural system dysfunction in schizophrenia more generally may be associated with developmental pathology 
due to a range of contributing factors, such as neuronal microcircuit abnormalities, altered synaptic plasticity mechanisms, impairments in activity-dependent inputs to the cortex, or some combination of these (Stephan et al, 2009). One proposal is that maladaptive experiencedependent plasticity due to impaired sensory processing may be contributing to disrupted neural integrity (Nithianantharajah and Hannan, 2006); this pathological process may therefore be an important treatment target that would be highly amenable to cognitive training treatment approaches (Vinogradov et al, 2012).

Consistent with this approach, intensive targeted cognitive training (TCT) of auditory processing and auditory working memory in people with schizophrenia has been found to improve cognition and generate neuroplastic changes in prefrontal and auditory cortices that are associated with behavioral gains (Popov et al, 2011; Subramaniam et al, 2014). Given its emphasis on training auditory perceptual processing and encoding (Fisher et al, 2009), and the association of auditory psychophysical improvement with generalized cognitive gains (Biagianti et al, 2016), it is highly likely that TCT relies heavily on thalamic sensory integration functions in order to drive improvements in higher-order cognitive abilities. Indeed, magnetoencephalography during a simple early auditory processing task reveals that $50 \mathrm{~h}$ of TCT evokes increases in high-gamma oscillatory activity in the prefrontal cortex that are significantly associated with gains in M100 amplitude in the primary auditory cortex; these changes are in turn related to improvements in an untrained executive functioning task (Dale et al, 2015). These findings support the notion that training-induced auditory cortical plasticity is yoked to adaptive plastic changes sourcelocalized to the prefrontal cortex, presumably through circuits involving thalamic processing of sensory inputs and cognition-action outputs. Additionally, a meta-analysis of cognitive training for schizophrenia more broadly (ie across all types of computerized and behavioral interventions) showed that these interventions evoke task-related functional activation increases in the thalamus (Ramsay and MacDonald, 2015), indicative of neural target engagement by the intervention.

Despite these early clues suggesting a critical role of the thalamus in the response to cognitive training interventions, no study to date has sought to determine whether intensive training is associated with changes in structural morphology of the thalamus that covary with cognitive improvement. Previous work has established that learning-induced plasticity can influence the structure and function of the human brain in as little as 3 months (Draganski et al, 2004, 2006), and morphometric changes in response to neural stimulation have been observed in as early as 5 days (May et al, 2007). Cognitive training in healthy young adults has been shown to result in increased structural gray matter in both frontal and posterior cortical brain areas (Ceccarelli et al, 2009), while in schizophrenia it has been shown that a 2 -year multimodal social and cognitive intervention was neuroprotective against gray matter loss in subcortical structures associated with emotional processing (Eack et al, 2010). However, no study to date has investigated whether cognitive training improvements in schizophrenia are associated with volumetric gains in brain areas that govern sensory integration, or whether such changes can occur after a relatively short-time period, particularly in young people in the earliest phases of the illness. Such findings would potentially be of high theoretical and clinical interest, given that the early phases of schizophrenia are associated with gray matter volume loss that appears to predict poor functional outcome (Cahn et al, 2006).

In this study of individuals early in the course of schizophrenia (ESZ), we sought to answer the following questions: (1) Do we observe changes in thalamic volume after an intensive course of $40 \mathrm{~h}$ of TCT of the auditory system, over an 8-10-week period? (2) Are any observed changes in thalamic volume significantly associated with TCT-induced improvements in cognition? (3) What are the baseline clinical, cognitive, and brain structure characteristics predictive of cognitive gains in response to TCT? We obtained baseline structural magnetic resonance imaging (MRI), as well as cognitive and clinical assessments, from a subset of ESZ patients who had participated in a randomized controlled trial of remotely delivered TCT (Fisher et al, 2014; Loewy et al, 2016). We examined thalamic volumes before and after TCT treatment (relative to a CG control condition), and hypothesized that cognitive response to TCT would be correlated with morphological changes in the thalamus.

\section{MATERIALS AND METHODS}

\section{Participants}

Participants in the current study were drawn from a clinical trial that was previously reported (Fisher et al, 2014) (Clinicaltrials.gov NTC00694889). ESZ participants were recruited from the Early Psychosis Clinic at the University of California, San Francisco and other community clinics. Participants were recruited via flyers, presentations, and clinician referrals, and were required to meet the following inclusion/exclusion criteria: (1) diagnosis of schizophrenia or schizoaffective disorder, confirmed using the Structured Clinical Interview for DSM-IV (SCID) (First et al, 1997); (2) onset of first psychotic episode within the last 5 years $(\mathrm{M}=1.68 ; \mathrm{SD}=1.36)$; (3) good general physical health; (4) age between 14 and 36 years old; (5) fluent and proficient in English; (6) IQ of 70 or greater; (7) no known neurological disorder; (8) no substance dependence in the past year; and (9) no MRI contraindications. All eligible participants had achieved outpatient status for at least 3 months, and were stable on psychiatric medications (Chlorpromazine equivalents (CPZ) were calculated based on haloperidol dose-year (Andreasen et al, 2010)). Participants 18 and older provided informed consent, while participants under 18 provided assent as well as parent or legal guardian consent. All consenting and baseline assessment procedures were conducted prior to random assignment (stratified by age, IQ, gender, and symptom severity) to one of the two training conditions. The Institutional Review Board at the University of California, San Francisco approved all study procedures.

\section{Training Procedures}

As previously described (Fisher et al, 2014), all participants were loaned a laptop to complete either the TCT $(N=22)$ or CG $(N=22)$ control intervention at home, and were asked to participate for $40 \mathrm{~h}$ over the course of 8 weeks ( $1 \mathrm{~h} /$ day, 
5 days/week). Each participant was contacted 1-2 times per week by telephone to discuss their progress, and coaching (ie, goal-setting, setting reminders) was provided if participants indicated difficulty maintaining their schedule. Check-in appointments were also conducted after every 10 sessions, where participants were paid $\$ 5$ for each completed hour, an additional $\$ 20$ for 10 completed sessions, and $\$ 30$ after completing $40 \mathrm{~h}$ of training. Participants also received $\$ 20$ for each assessment appointment.

TCT was provided by Posit Science, and consisted of adaptive computerized exercises designed to improve the speed and accuracy of early auditory processing while engaging in auditory and verbal working memory tasks (Fisher et al, 2009). Such tasks seek to target early sensory processes that rely on thalamic integration and connectivity with prefrontal and auditory cortices to carry out higher-order auditory and cognitive processes (Lee et al, 2013). TCT exercises were individually adapted in difficulty level to maintain $80-85 \%$ accuracy. In each session, participants completed four to six exercises over the course of $1 \mathrm{~h}$. Exercises initially focused on basic sensory processes, such as phoneme distinction and frequency modulation sweeps, before progressing onto more complicated tasks of auditory working memory, requiring adequate integration of more basic sensory processing skills. Correct trials on these tasks were rewarded with points and small animations. Participant compliance was monitored remotely via electronic data upload.

The CG control condition was matched to the TCT condition for computer exposure, contact with research personnel, monetary incentives, and nonspecific engagement of attention, executive functioning, and motivation (embedded within the CG). CG participants played 16 different commercially available CG, playing 4-5 different games per training day. Examples of games included Solitaire, Freecell, and Pinball. Participants completed an average of 36.64 $(\mathrm{SD}=7.07)$ hours of training in the TCT condition, and $39.82(\mathrm{SD}=.59)$ hours in the CG condition $(t=-2.10$; $p=0.05$ ). Number of days to complete training (and subsequent time between scans) did not differ between the TCT $(M=131.95 ; S D=53.81)$ and CG $(M=142.00 ; S D=$ $57.85)$ conditions $(t=-0.59 ; p=0.56)$.

\section{Assessment Procedures}

Assessments were conducted blind to treatment group assignment. Symptoms were assessed with the Positive and Negative Syndrome Scale (PANSS)(Kay et al, 1987), while cognition was assessed using an abbreviated battery of MATRICS-recommended measures (Nuechterlein et al, 2008) (Trail Making Test Part A; Animal Fluency; LetterNumber Span; Wechsler Memory Scale-III Spatial Span; Hopkins Verbal Learning Test-Revised; Brief Visuospatial Memory Test-Revised; Delis-Kaplan Executive Functioning System Tower Test). All MATRICS subtests were converted to age-normed $Z$-scores before taking the average $Z$-score across all subtests to calculate the Global Cognition summary score.

\section{Neuroimaging Procedures and Pre-Processing}

Structural MRI was obtained at baseline, and after completion of the intervention (mean of 19.58 weeks, SD of
7.93 weeks). Whole brain volumetric magnetizationprepared rapid gradient echo (MPRAGE) scans were acquired with $\mathrm{TR}=2300 \mathrm{~ms}$, TE $=2.95 \mathrm{~ms}$, TI $=900 \mathrm{~ms}$, Flip Angle $=9^{\circ}$, Base Resolution $=256$, Slice Thickness $=1.2 \mathrm{~mm}$, and anterior to posterior collection in the sagittal plane. Subcortical segmentation was generated using FreeSurfer (v5.1), a software program used to process and analyze brain MRI images (Fischl et al, 1999; Fischl and Dale, 2000). FreeSurfer carries out Talairach transformations, atlas registration, subcortical white matter and gray matter segmentation, tessellation of gray and white matter boundaries, and placing borders around gray/white matter and gray/cerebrospinal fluid boundaries based on an intensity gradient. Following this step, each image was manually inspected for segmentation errors and was corrected to ensure that the white/gray matter boundaries were accurately captured. This process was applied separately to data from each time-point in order to remove the possibility of a temporal bias.

Next, images were passed through the Freesurfer longitudinal volumetric pipeline (Reuter et al, 2012), where a within-subject template (base) was created by averaging the two time-points together in order to estimate the average anatomy of the subject across time based on the intensity median of each voxel. The base was then manually checked for registration and overlay errors. Each time-point was then processed longitudinally, and automatically compared to the base. Each longitudinal time-point was manually checked for overlay, registration, and boundary errors. Finally, measurements were obtained from the processed images for the left and right thalamic regions of interest based on parcellation data from the Desikan-Killany atlas (Desikan et al, 2006).

\section{Planned Analyses}

Pre- $v s$ post-training global cognition scores were converted to $Z$-scores and compared between groups in a repeated measures ANOVA. Changes in thalamic volume were compared from pre- to post-training between groups, both examining raw thalamic volume changes in a repeated measures ANOVA, as well as in a repeated measures ANCOVA co-varying for both baseline age and intracranial volume. Next, we used a linear regression to examine the relationship between change in cognition (post-pre) and change in thalamic volume (post-pre), and whether the regression slopes of these relationships differed between TCT and CG. We followed up on these analyses with relevant Pearson correlation tests where appropriate. Next we used a linear regression to determine whether the regression slopes differed between groups predicting changes in cognition on the basis of baseline symptoms (measured by PANSS positive, negative, and global scores), baseline thalamic volume, and baseline global cognition. Last, we used linear regression to determine whether regression slopes differed between groups predicting changes in thalamic volume on the basis of baseline symptoms, baseline thalamic volume, and baseline global cognition. Again, we followed up with Pearson correlation tests where appropriate. 


\section{RESULTS}

Forty-four individuals who were randomized and completed 20-40 h of scanning underwent MRI scanning (TCT =22; $\mathrm{CG}=22$; see Supplementary CONSORT Diagram). The TCT and CG conditions did not differ on baseline factors related to demographic, clinical, cognitive, or thalamic volume (all p's >.10; see Table 1). Behavioral results examining global cognition comparing pre- $v s$ post-training did not show within- or between-group differences $(p>0.25$; see Table 2$)$, and the between-group effect size was modest $(d=0.33)$. Similarly, neither left nor right thalamus showed within- or between-group differences ( $p$ 's $>0.40$; see Table 2 ), with a small between-group effect size in the left thalamus $(d=0.25)$ as well as in the right thalamus $(d=-0.11)$. This was found to be the case both with and without covariation for intracranial volume and age. Next, we examined whether baseline thalamic volume correlated with baseline neurocognition. Left thalamic volume showed a significant correlation with neurocognition $(r=0.30 ; p=0.04$; Figure $1 \mathrm{a})$, while the right thalamus showed a similar, though nonsignificant relationship ( $r=0.25 ; p=0.10$; Figure $1 b)$.

Next, we sought to understand the relationship between changes in global cognition and change in thalamic volume. We entered thalamic volume change (both left and right hemispheres), group, and their interaction in a linear mixed model predicting change in cognition. This analysis demonstrated a significant group $\times$ thalamic volume change interaction $\left(\chi^{2} \quad(1)=13.62, p=0.0002\right)$. To more closely interrogate this effect in individual hemispheres, group, left thalamic volume change, and their interaction were entered into a linear regression model as predictors of change in cognition. A significant group $\times$ thalamic volume change interaction emerged $(t=3.88 ; p=0.0004$; Figure $1 \mathrm{c})$, indicating a significant difference in slopes between the groups. These results held when controlling for baseline cognition $(t=3.99 ; p=0.0003)$. We followed up on this finding to examine each group individually. Improvements in cognition significantly correlated with change in left thalamic volume in the TCT group $(r=0.62 ; p=0.002)$, while this positive relationship was not observed in the CG condition; in fact there was a statistical trend toward the opposite pattern $(r=-0.40 ; p=0.06)$. Again, these results were maintained when conducting partial correlations controlling for baseline cognition in both groups. We repeated this analysis in the right thalamus and showed no significant difference in slopes $(t=1.46 ; p=0.15)$ and no significant common slope across groups $(t=1.40 ; p=0.14)$, though nonsignificant patterns were similar to the significant effects observed in the left thalamus, in that change in the right thalamic volume had a positive relationship with cognitive improvements in response to TCT $(r=0.38 ; p=0.08)$, and a modestly negative relationship in the CG condition $(r=-0.05 ; p=0.83)$.

Last we sought to examine whether baseline measures were predictive of response to training or changes in thalamic volume. Group, baseline symptom severity measured by the positive, negative, and general PANSS subscales, and their interactions were entered into linear regression models as predictors of overall cognitive change (see Table 3). We did not observe significant group $\times$ baseline symptom interactions for either positive $(t=0.24 ; p=0.98)$ or negative symptom subscales $(t=1.60 ; p=0.12)$, but did observe a
Table I Baseline Demographic, Cognitive, Clinical, and Thalamic Volume Scores

\begin{tabular}{|c|c|c|c|c|c|c|}
\hline & \multicolumn{2}{|c|}{$\begin{array}{c}\text { TCT }(N=22 ; \\
\text { males }=14)\end{array}$} & \multicolumn{2}{|c|}{$\begin{array}{l}\text { CG }(N=22 ; \\
\text { males = I5) }\end{array}$} & \multirow[b]{2}{*}{ t-Value } & \multirow[b]{2}{*}{$p$-Value } \\
\hline & Mean & SD & Mean & SD & & \\
\hline Age (years) & 23.28 & 4.57 & 21.25 & 3.68 & 1.62 & 0.11 \\
\hline $\begin{array}{l}\text { Duration of illness } \\
\text { (years) }\end{array}$ & 1.68 & 1.22 & 1.71 & 1.38 & -0.09 & 0.93 \\
\hline Education (years) & 12.73 & 1.86 & 12.55 & 3.21 & 0.22 & 0.82 \\
\hline $\begin{array}{l}\text { Verbal IQ } \\
\text { (standard score) }\end{array}$ & 105.73 & 13.94 & 103.50 & 15.33 & 0.50 & 0.62 \\
\hline $\begin{array}{l}\text { Left thalamus } \\
\text { (cubic ml) }\end{array}$ & 7332.05 & 971.00 & 7487.77 & 804.08 & -0.58 & 0.57 \\
\hline $\begin{array}{l}\text { Right thalamus } \\
\text { (cubic ml) }\end{array}$ & 7460.22 & | 108.65 & 7728.18 & 902.96 & -0.88 & 0.38 \\
\hline $\begin{array}{l}\text { Neurocognition } \\
\text { (Z-score) }\end{array}$ & -1.01 & 0.81 & -0.77 & 0.70 & -1.07 & 0.29 \\
\hline PANSS total & 60.54 & $|1.4|$ & $65.7 \mid$ & 18.82 & -1.08 & 0.29 \\
\hline PANSS general & 31.82 & 6.88 & 34.81 & 10.83 & -1.08 & 0.29 \\
\hline PANSS positive & 12.05 & 3.42 & 13.24 & 4.76 & -0.94 & 0.35 \\
\hline PANSS negative & 16.68 & 6.48 & 17.67 & 7.17 & -0.47 & 0.64 \\
\hline $\begin{array}{l}\text { Medication } \\
\text { (CPZ equivalence) }\end{array}$ & 361.11 & 456.56 & 387.08 & 346.47 & -0.16 & 0.87 \\
\hline
\end{tabular}

Abbreviations: CG, computer games; CPZ, chlorpromazine equivalents; IQ, intelligence quotient; PANSS, Positive and Negative Symptom Scale; TCT, targeted cognitive training.

significant difference in slopes between groups for the general symptom subscale $(t=-2.04 ; p=0.048)$. Lower baseline general symptoms were found to more strongly predict overall cognitive improvement in the TCT group $(r=-0.59$; $p=0.004)$ compared with the CG group $(r=-0.42 ; p=0.06)$.

To determine whether baseline symptoms were predictive of morphological changes, group, baseline symptom severity, and their interactions were entered into linear regression models as predictors of left thalamic volume change (see Table 3). Though positive symptoms did not show a group $\times$ baseline symptom interaction $(t=0.11 ; p=0.92)$, we did observe significant interactions for both negative $(t=-2.58 ; p=0.01)$ and general baseline symptoms $(t=-2.23 ; p=0.03)$, again indicating slope differences between TCT and CG. Lower baseline negative symptoms related to increased left thalamic volume in the TCT group $(r=-0.36$; $p=0.10)$, while the opposite pattern was observed in the CG group $(r=0.41 ; p=0.07)$. Similarly, lower baseline general symptoms were related to increased left thalamic volume in TCT $(r=-0.41 ; p=0.06)$, while the opposite was observed for CG $(r=0.20 ; p=0.39)$. No relationships between baseline thalamic volume and changes in overall cognition or changes in thalamic volume were observed ( $p$ 's $>0.55$ ). Additionally, no relationships between baseline overall cognition and changes in either cognition or thalamic volume were observed ( $p$ 's $>0.50$ ). None of these relationships were observed in the right thalamus ( $p$ 's $>0.72$ ), and none of the above relationships were found to change when covarying for age and intracranial volume. We also conducted post hoc analyses in other regions of interest, including the hippocampus, amygdala, nucleus accumbens, temporal gyrus, and middle frontal gyrus. No significant group $\times$ time volume effects and no significant correlations 
Table 2 Change Scores for Cognitive, Clinical, and Thalamic Volume

\begin{tabular}{|c|c|c|c|c|c|c|c|}
\hline & \multicolumn{2}{|c|}{ TCT } & \multicolumn{2}{|c|}{ CG } & \multirow[b]{2}{*}{ t-Value } & \multirow[b]{2}{*}{$p$-Value } & \multirow[b]{2}{*}{ Effect size ( $d$ ) } \\
\hline & Mean & SD & Mean & SD & & & \\
\hline Right thalamus (cubic ml) & -5.73 & 117.89 & 5.41 & 97.38 & -0.34 & 0.73 & -0.10 \\
\hline Neurocognition (Z-score) & 0.18 & 0.59 & 0.01 & 0.40 & 1.14 & 0.26 & 0.33 \\
\hline PANSS positive & -1.25 & 2.86 & -2.17 & 3.60 & 0.77 & 0.45 & 0.28 \\
\hline PANSS negative & -1.75 & 5.34 & 1.11 & 6.23 & -1.34 & 0.19 & -0.49 \\
\hline
\end{tabular}

Abbreviations: CG, computer games; Neurocognition, Global Cognition Score; PANSS, Positive and Negative Symptom Scale; TCT, targeted cognitive training. Effect size was calculated using Cohen's $d$.
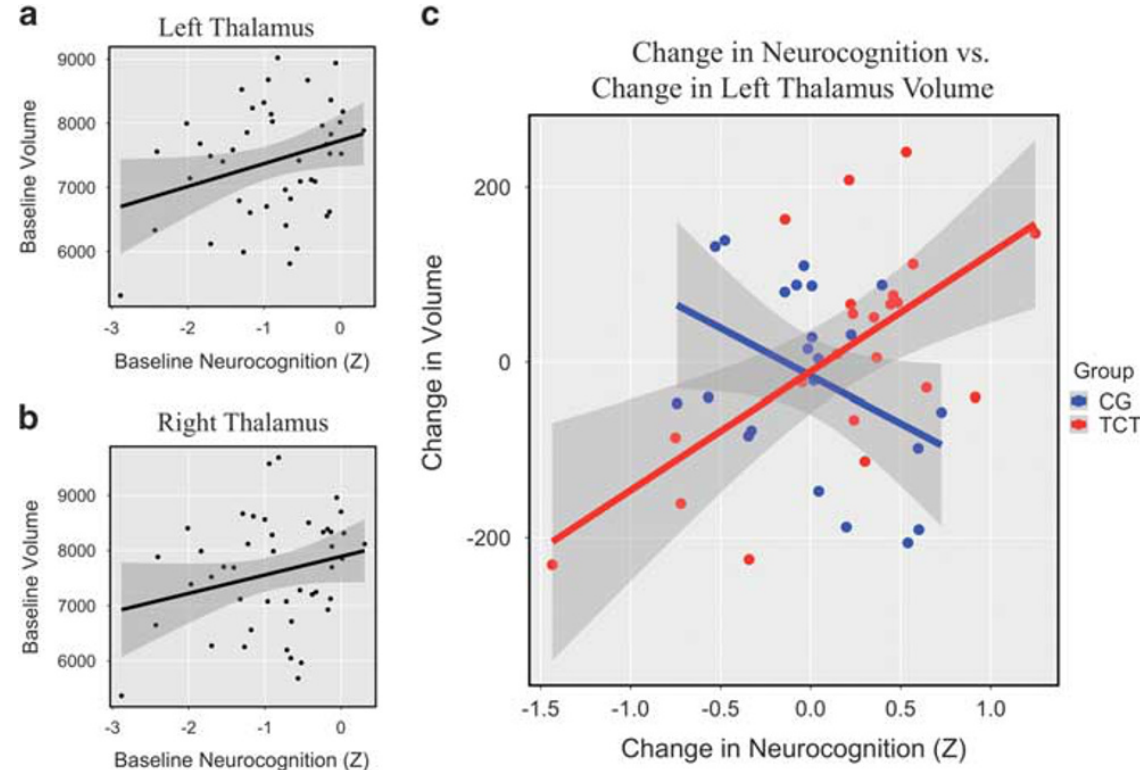

Figure I (a) Baseline left thalamic volume (cubic ml) correlated with baseline neurocognition $(r=0.30$; $p<0.05$ ). (b) Baseline right thalamic volume (cubic $\mathrm{ml}$ ) showed a similar, though nonsignificant relationship with baseline neurocognition $(r=0.25 ; p=0.10)$. (c) The difference in slopes between TCT and CG was significant $(t=3.88 ; p<0.0005)$. Increases in left thalamic volume (cubic $\mathrm{ml}$ ) significantly correlated with improvements in neurocognition in the TCT condition $(r=0.62 ; p<0.005)$, while reduced left thalamic volume showed a trend with increases in cognition in the CG condition $(r=-0.40 ; p=0.06)$. Shaded area indicates $95 \%$ confidence interval.

between change in volume and change in cognition were observed (see Supplementary Table).

\section{DISCUSSION}

We found that changes in left thalamic volume were positively correlated to changes in neurocognitive outcome scores following $40 \mathrm{~h}$ (over 8-10 weeks) of TCT of auditory perceptual processing and working memory delivered remotely to young individuals with ESZ, but not the CG control condition. Though we did not observe significant morphological changes at a group level in response to training (no significant group $\times$ time effects), individuals with ESZ who showed cognitive improvement in response to remotely delivered TCT showed increases in left thalamic volume over the $\sim 4$ months assessment period. Left thalamic volume was also significantly correlated with global cognition at baseline. Notwithstanding the limitations of what can be concluded from correlational analysis, these results suggest that in early phases of schizophrenia, TCT may induce experience-dependent structural plasticity in the thalamus and associated cognitive improvement in a manner not observed in the control condition. It is unclear why we observe a trend toward the opposite pattern in the CG condition, but it possibly suggests that sustained nontargeted activities (ie simple CG) evoke structural changes that could have deleterious influence on patients with schizophrenia. Previous studies have shown reduced verbal learning and memory following CG (Fisher et al, 2009, 2014), which may also be undergirded by changes in neurobiological structure and function, though this relationship was not observed in the current study.

Preservation and enhancement of thalamic volume in ESZ is likely to be of high clinical importance, especially as 
Table 3 Linear Regressions Predicting Cognitive and Left Thalamic Volume Changes on the Basis of Group (TCT vs CG) and Baseline Symptoms from the PANSS (Positive, Negative, and General Symptom Scores)

\begin{tabular}{|c|c|c|c|c|c|}
\hline Dependent variable & Independent variables & Estimate & Standard error & t-Value & p-Value \\
\hline \multirow[t]{3}{*}{ Cognitive change } & Group & 261.22 & 93.791 & 2.785 & 0.0082 \\
\hline & Volume change & 6.048 & 3.465 & 1.746 & 0.0088 \\
\hline & Group $\times$ Volume change & 0.0044 & 0.0011 & 3.884 & 0.0004 \\
\hline \multirow[t]{2}{*}{ Cognitive change } & Group & 0.1151 & 0.5164 & 0.223 & 0.825 \\
\hline & Baseline positive $\mathrm{S} x$ & -0.0368 & 0.0234 & -1.573 & 0.124 \\
\hline \multirow[t]{3}{*}{ Cognitive change } & Group & -0.4656 & 0.4192 & -1.111 & 0.2736 \\
\hline & Baseline negative Sx & -0.03101 & 0.0155 & -2.002 & 0.0522 \\
\hline & Group $\times$ Baseline Negative Sx & 0.0363 & 0.0228 & $|.59|$ & 0.1196 \\
\hline \multirow[t]{2}{*}{ Cognitive change } & Group & 1.2014 & 0.5618 & 2.138 & 0.0388 \\
\hline & Baseline general $\mathrm{S} x$ & -0.016 & 0.0091 & $-1.76 \mid$ & 0.086 \\
\hline \multirow[t]{3}{*}{ Volume change } & Group & 261.221 & 93.791 & 2.785 & 0.0082 \\
\hline & Baseline negative $\mathrm{S} x$ & 6.048 & 3.465 & 1.746 & 0.0888 \\
\hline & Group $\times$ Baseline Negative Sx & -13.179 & 5.101 & -2.584 & 0.01364 \\
\hline \multirow[t]{3}{*}{ Volume change } & Group & 344.576 & | 44.168 & 2.39 & 0.0218 \\
\hline & Baseline general Sx & 1.926 & 2.332 & 0.826 & 0.4138 \\
\hline & Group × Baseline General Sx & -9.535 & 4.276 & -2.23 & 0.0316 \\
\hline
\end{tabular}

disrupted thalamic integrity is found to relate to structural abnormalities observed across the cortex (Cobia et al, 2016), in addition to poor clinical outcomes (Brickman et al, 2004). As such, an intervention targeting thalamic integrity may be able to systematically rescue or reverse the cognitive problems found to be pervasive in schizophrenia. Exercising auditory sensory processing and working memory through TCT has previously been shown to influence functional plasticity in both auditory and prefrontal cortical areas (Dale et al, 2015; Subramaniam et al, 2014). Of note, the thalamus is well understood to project both directly and indirectly to the prefrontal cortex by way of the medial dorsal nucleus (Miller and Cohen, 2001), and is therefore implicated in a range of higher-order cognitive abilities. Functional thalamoprefrontal connections are disrupted in schizophrenia (Giraldo-chica and Woodward, 2016), but have also been shown to be malleable in response to cognitive training, with reversal of these disruptions coinciding with improvements in global cognition (Ramsay et al, 2016). In light of the current findings, this may suggest that cognitive training works by targeting sensory processes that exercise and maintain thalamic integrity, thereby promoting thalamocortical connections that underlie higher order cognition and functioning. We also note that these relationships were not strictly driven by improvements in working memory, as post hoc correlation analyses removing working memory from the global cognition score were still shown to be significantly related to change in left thalamic volume $(r=0.60 ; p=0.003)$.

We also find that ESZ patients with lower general psychiatric symptom severity at baseline were more likely to improve on outcome measures of global cognition.
Additionally, both lower negative and general symptoms at baseline related to either preservation or increased thalamic left thalamic volume, in response to TCT. This effect was not observed in the control condition, and is consistent with previous behavioral meta-analytic findings showing that lower baseline symptoms are related to global cognitive improvements following treatment (Wykes et al, 2011). This indicates that response to treatment, both with regard to gains in cognition and volumetric changes, may be driven by baseline factors related to illness severity in schizophrenia. Though we did not observe a relationship between chlorpromazine equivalents and TCT response, it is likely that individuals with less severe symptoms are more able to engage with the cognitive training regimen; they may also have a form of the illness characterized by less severe impairments in inherent neuroplasticity capacities. We did not find that baseline global cognition or baseline thalamic volume was predictive of treatment response.

Though improved global cognition was positively correlated with changes in thalamic volume in the TCT group and not in the control group, we did not observe a treatmentdriven group-by-time interaction effect in either global cognition or thalamic volume. While a reasonably large effect on global cognition $(d=0.74)$ in response to TCT was observed in the full randomized trial (Fisher et al, 2014), we observed only a moderate effect $(d=0.33)$ in the subsample that participated in the MRI; this may have reduced our ability to observe a group-by-time effect in the thalamus ROI. Relatedly, not all TCT participants completed the full $40 \mathrm{~h}$ of training, and though we did not observe any relationships between duration of training and cognitive 
outcome, this may have weakened the present effects. It is also noteworthy that significant relationships between thalamic volume and both baseline global cognition and change in global cognition were more robustly observed in the left hemisphere. Though statistical trends were observed in the right thalamus, it is unclear whether a larger sample would reveal a bilateral influence of TCT, or if the left hemisphere is more amenable to plasticity due to the auditory and verbal nature of the intervention (Belin et al, 1998).

While the thalamus appears to be a critically important treatment target, we also followed up with post hoc analyses in other cortical and subcortical brain areas hypothesized to be implicated in cognitive training for schizophrenia. In a recent multi-site study, subcortical volume reduction was observed in the thalamus in addition to the hippocampus, amygdala, and the nucleus accumbens (van Erp et al, 2016). No group $\times$ time effects or correlations with changes in cognition was found to be significant. We also followed up on auditory and prefrontal cortical regions previously shown to exhibit functional changes in response to TCT (Dale et al, 2015; Subramaniam et al, 2014). Again, none were found to be significant.

Based on these post hoc analyses, it is unclear whether TCT-induced morphological changes are simply the result of focal changes in the thalamus, or if the observed changes in cognition may also be dependent on structural or functional changes in cortical regions as well. Overall, this is a limitation of the current study, and further work will be required to integrate the observed thalamic changes with findings indicating that TCT evokes functional plasticity in prefrontal and temporal cortices (Dale et al, 2015). Relatedly, the current study was limited in its ability to examine subthalamic nuclei that may be driving the observed thalamic volume changes, and may subsequently relate more directly to changes in overall cognition. Regions including the medial dorsal nucleus of the thalamus projects to the prefrontal cortex, while the medial genticulate nucleus of the thalamus projects to the auditory cortex (Behrens et al, 2003); notably, these thalamic nuclei are understood to be involved in perception, attention, and higher-order cognition. Changes in thalamic subregions are likely implicated in the improvements associated with TCT, but remain challenging to directly measure with available segmentation tools (Keller et al, 2012). Continued work and novel methods, possibly combining diffusion and volumetric metrics, will be required to clarify the relationship between thalamic nuclei plasticity and cognitive changes induced by TCT.

In sum, the current study demonstrates that cognitive gains in response to TCT are positively correlated with changes in thalamic volume in ESZ occurring over a 4-month period, and that less severe symptoms prior to training are predictive of this response. This suggests that TCT may repair or slow progressive brain pathology associated with early phases of the illness, and that it does so in a brain region critical for successful higher-order cognition. Future work will be required to determine whether these findings are replicable in a larger sample (as this and other studies have only modest sample sizes), and to establish their long-term cognitive and functional implications for individual patients.

\section{FUNDING AND DISCLOSURE}

The current study was funded by the Stanley Medical Research Institute (06TAF-972) and the National Institute of Mental Health (MH076989). ISR was funded by the Wells Family Trust. The training software used in this study was supplied free of charge by Posit Science. SV has also served as a paid consultant to Posit Science Inc. Within the past three years, DHM has received compensation as a consultant for Boehringer-Ingelheim, Amgen, and Hoffmann-LaRoche. The remaining authors declare no conflict of interest.

\section{REFERENCES}

Andreasen NC, Arndt S, Swayze V, Cizadlo T, Flaum M, O’Leary D et al (1994). Thalamic abnormalities in schizophrenia visualized through magnetic resonance image averaging. Science 266: 294-298.

Andreasen NC, Pressler M, Nopoulos P, Miller D, Ho BC (2010). Antipsychotic dose equivalents and dose-years: a standardized method for comparing exposure to different drugs. Biol Psychiatry 67: 255-262.

Behrens TEJ, Johansen-Berg H, Woolrich MW, Smith SM, WheelerKingshott CAM, Boulby PA et al (2003). Non-invasive mapping of connections between human thalamus and cortex using diffusion imaging. Nat Neurosci 6: 750-757.

Belin P, Zilbovicius M, Crozier S, Thivard L, Fontaine A, Masure M-C et al (1998). Lateralization of speech and auditory temporal processing. J Cogn Neurosci 10: 536-540.

Biagianti B, Fisher M, Neilands TB, Loewy R, Vinogradov S (2016). Engagement with the auditory processing system during targeted auditory cognitive training mediates changes in cognitive outcomes in individuals with schizophrenia. Neuropsychology 30: 998-1008.

Brickman AM, Buchsbaum MS, Shihabuddin L, Byne W, Newmark $\mathrm{RE}$, Brand $\mathrm{J}$ et al (2004). Thalamus size and outcome in schizophrenia. Schizophr Res 71: 473-484.

Cahn W, Van Haren NEM, Hulshoff Pol HE, Schnack HG, Caspers E, Laponder DAJ et al (2006). Brain volume changes in the first year of illness and 5-year outcome of schizophrenia. Br J Psychiatry 189: 381-382.

Ceccarelli A, Rocca MA, Pagani E, Falini A, Comi G, Filippi M (2009). Cognitive learning is associated with gray matter changes in healthy human individuals: a tensor-based morphometry study. Neuroimage 48: 585-589.

Çetin MS, Christensen F, Abbott CC, Stephen JM, Mayer AR, Cañive JM et al (2014). Thalamus and posterior temporal lobe show greater inter-network connectivity at rest and across sensory paradigms in schizophrenia. Neuroimage 97: 117-126.

Cobia DJ, Smith MJ, Salinas I, Ng C, Gado M, Csernansky JG et al (2016). Progressive deterioration of thalamic nuclei relates to cortical network decline in schizophrenia. Schizophr Res 180: 21-27.

Cronenwett WJ, Csernansky J (2010). Thalamic pathology in schizophrenia. Curr Top Behav Neurosci 4: 509-528.

Dale CL, Brown EG, Fisher M, Herman AB, Dowling AF, Hinkley LB et al (2015). Auditory cortical plasticity drives training-induced cognitive changes in schizophrenia. Schizophr Bull 42: 1-9.

Desikan RS, Ségonne F, Fischl B, Quinn BT, Dickerson BC, Blacker D et al (2006). An automated labeling system for subdividing the human cerebral cortex on MRI scans into gyral based regions of interest. Neuroimage 31: 968-980.

Doniger GM, Silipo G, Rabinowicz EF, Snodgrass JG, Javitt DC (2001). Impaired sensory processing as a basis for object-recognition deficits in schizophrenia. Am J Psychiatry 158: 1818-1826.

Draganski B, Gaser C, Busch V, Schuierer G, Bogdahn U, May A (2004). Neuroplasticity: changes in grey matter induced by training. Nature 427: 311-312.

Draganski B, Gaser C, Kempermann G, Kuhn HG, Winkler J, Buchel $C$ et al (2006). Temporal and spatial dynamics of brain structure changes during extensive learning. J Neurosci 26: 6314-6317. 
Eack SM, Hogarty GE, Cho RY, Prasad KMR, Greenwald DP, Hogarty SS et al (2010). Neuroprotective effects of cognitive enhancement therapy against gray matter loss in early schizophrenia: results from a 2-year randomized controlled trial. Arch Gen Psychiatry 67: 674-682.

Ellison-Wright I, Glahn DC, Laird AR, Thelen SM, Bullmore E (2008). The anatomy of first-episode and chronic schizophrenia: an anatomical likelihood estimation meta-analysis. Am J Psychiatry 165: 1015-1023. van Erp TGM, Hibar DP, Rasmussen JM, Glahn DC, Pearlson GD, Andreassen OA et al (2016). Subcortical brain volume abnormalities in 2028 individuals with schizophrenia and 2540 healthy controls via the ENIGMA consortium. Mol Psychiatry 21: 547-553.

First MB, Spitzer RL, Gibbon M, Williams JBW (1997). Structured Clinical Interview for DSM-IV Axis I Disorders, Clinician Version (SCID-CV). for DSMIV. https://scholar.google.es/scholar?q=re lated:XNMzPEvvkzYJ:scholar.google.com/\&hl=es\&as_sdt=0,33.

Fischl B, Dale AM (2000). Measuring the thickness of the human cerebral cortex from magnetic resonance images. Proc Natl Acad Sci 97: 11050-11055.

Fischl B, Sereno MI, Dale AM (1999). Cortical surface-based analysis: II: Inflation, flattening, and a surface-based coordinate system. Neuroimage 9: 195-207.

Fisher M, Holland C, Merzenich MM, Vinogradov S (2009). Using neuroplasticity-based auditory training to improve verbal memory in schizophrenia. Am J Psychiatry 166: 805-811.

Fisher M, Loewy R, Carter C, Lee A, Ragland JD, Niendam T et al (2014). Neuroplasticity-based auditory training via laptop computer improves cognition in young individuals with recent onset schizophrenia. Schizophr Bull 41: 1-9.

Gilbert CD, Sigman M (2007). Brain states: top-down influences in sensory processing. Neuron 54: 677-696.

Giraldo-chica M, Woodward ND (2016). Review of thalamocortical resting-state fMRI studies in schizophrenia. Schizophr Res 180: 6-11.

Guillery RW (1995). Anatomical evidence concerning the role of the thalamus in corticocortical communication: a brief review. J Anat 187(Pt 3): 583-592.

Haren NEM van, Pol HEH, Schnack HG, Cahn W, Mandl RCW, Collins DL et al (2007). Focal gray matter changes in schizophrenia across the course of the illness: a 5-year followup study. Neuropsychopharmacology 32: 2057-2066.

Ho B-C, Andreasen NC, Ziebell S, Pierson R, Magnotta V (2011). Longterm antipsychotic treatment and brain volumes: a longitudinal study of first-episode schizophrenia. Arch Gen Psychiatry 68: 128-137.

Javitt DC (2009). Sensory processing in schizophrenia: neither simple nor intact. Schizophr Bull 35: 1059-1064.

Kay SR, Fiszbein A, Opler LA (1987). The positive and negative syndrome scale (PANSS) for schizophrenia. Schizophr Bull 13: 261-276.

Keller SS, Gerdes JS, Mohammadi S, Kellinghaus C, Kugel H, Deppe K et al (2012). Volume estimation of the thalamus using FreeSurfer and stereology: consistency between methods. Neuroinformatics 10: 341-350.

Konick LC, Friedman L (2001). Meta-analysis of thalamic size in schizophrenia. Biol Psychiatry 49: 28-38.

Lawrie SM, Whalley HC, Abukmeil SS, Kestelman JN, Donnelly L, Miller P et al (2001). Brain structure, genetic liability, and psychotic symptoms in subjects at high risk of developing schizophrenia. Biol Psychiatry 49: 811-823.

Lee RSC, Redoblado-Hodge MA, Naismith SL, Hermens DF, Porter Ma, Hickie IB (2013). Cognitive remediation improves memory and psychosocial functioning in first-episode psychiatric outpatients. Psychol Med 43: 1161-1173.

Light GA, Swerdlow NR, Braff DL (2007). Preattentive sensory processing as indexed by the MMN and $\mathrm{P} 3 \mathrm{a}$ brain responses is associated with cognitive and psychosocial functioning in healthy adults. J Cogn Neurosci 19: 1624-1632.
Loewy R, Fisher M, Schlosser DA, Biagianti B, Stuart B, Mathalon $\mathrm{DH}$ et al (2016). Intensive auditory cognitive training improves verbal memory in adolescents and young adults at clinical high risk for psychosis. Schizophr Bull 42: S118-S126.

May A, Hajak G, Gänßbauer S, Steffens T, Langguth B, Kleinjung T et al (2007). Structural brain alterations following 5 days of intervention: dynamic aspects of neuroplasticity. Cereb Cortex 17: 205-210.

Miller EK, Cohen JD (2001). An integrative theory of prefrontal cortex function. Neurosci Res 24: 167-202.

Morgenstern NA, Bourg J, Petreanu L (2016). Multilaminar networks of cortical neurons integrate common inputs from sensory thalamus. Nat Neurosci 19: 1034-1040.

Nithianantharajah J, Hannan AJ (2006). Enriched environments, experience-dependent plasticity and disorders of the nervous system. Nat Rev Neurosci 7: 697-709.

Nuechterlein KH, Green MF, Kern RS, Baade LE, Barch DM, Cohen JD et al (2008). The MATRICS consensus cognitive battery, Part 1: Test selection, reliability, and validity. Am J Psychiatry 165: 203-213.

Pergola G, Selvaggi P, Trizio S, Bertolino A, Blasi G (2015). The role of the thalamus in schizophrenia from a neuroimaging perspective. Neurosci Biobehav Rev 54: 1-19.

Pergola G, Trizio S, Di Carlo P, Taurisano P, Mancini M, Amoroso N et al (2017). Grey matter volume patterns in thalamic nuclei are associated with familial risk for schizophrenia. Schizophr Res 180: 13-20.

Popov T, Jordanov T, Rockstroh B, Elbert T, Merzenich MM, Miller GA (2011). Specific cognitive training normalizes auditory sensory gating in schizophrenia: a randomized trial. Biol Psychiatry 69: 465-471.

Ramsay IS, MacDonald III AW (2015). Brain correlates of cognitive remediation in schizophrenia: activation likelihood analysis shows preliminary evidence of neural target engagement. Schizophr Bull 41: 1276-1284.

Ramsay IS, Nienow TM, MacDonald AW (2016). Increases in intrinsic thalamocortical connectivity and overall cognition following cognitive remediation in chronic schizophrenia. Biol Psychiatry Cogn Neurosci Neuroimaging 2: 355-362.

Reuter M, Schmansky NJ, Rosas HD, Fischl B (2012). Withinsubject template estimation for unbiased longitudinal image analysis. Neuroimage 61: 1402-1418.

Saalmann YB, Kastner S (2011). Cognitive and perceptual functions of the visual thalamus. Neuron 71: 209-223.

Seidman LJ, Faraone SV, Goldstein JM, Goodman JM, Kremen WS, Toomey $\mathrm{R}$ et al (1999). Thalamic and amygdala-hippocampal volume reductions in first-degree relatives of patients with schizophrenia: an MRI-based morphometric analysis. Biol Psychiatry 46: 941-954.

Stephan KE, Friston KJ, Frith CD (2009). Dysconnection in schizophrenia: from abnormal synaptic plasticity to failures of self-monitoring. Schizophr Bull 35: 509-527.

Subramaniam K, Luks TL, Garrett C, Chung C, Fisher M, Nagarajan $S$ et al (2014). Intensive cognitive training in schizophrenia enhances working memory and associated prefrontal cortical efficiency in a manner that drives long-term functional gains. Neuroimage 99: 281-292.

Vinogradov S, Fisher M, de Villers-Sidani E (2012). Cognitive training for impaired neural systems in neuropsychiatric illness. Neuropsychopharmacology 37: 43-76.

Wykes T, Huddy V, Cellard C, McGurk SR, Czobor P (2011). A meta-analysis of cognitive remediation for schizophrenia: methodology and effect sizes. Am J Psychiatry 168: 472-485.

Yeap S, Kelly SP, Sehatpour P, Magno E, Garavan H, Thakore JH et al (2008). Visual sensory processing deficits in schizophrenia and their relationship to disease state. Eur Arch Psychiatry Clin Neurosci 258: 305-316.

Supplementary Information accompanies the paper on the Neuropsychopharmacology website (http://www.nature.com/npp) 